\title{
Can increased or decreased COX2 and P21 expression be used in illness monitoring of gastric cancer and its potential precursor lesions?
}

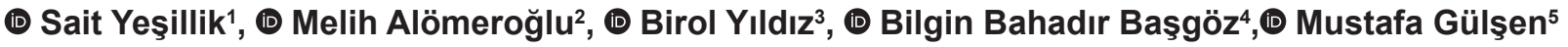 \\ 1 University of Health Sciences Turkey, Gülhane Training and Research Hospital, Clinic of Internal Medicine, Division of \\ Immunology and Allergy, Ankara, Turkey \\ 2University of Health Sciences Turkey, Gülhane Faculty of Medicine, Department of Pathology, Ankara, Turkey \\ 3University of Health Sciences Turkey, Gülhane Training and Research Hospital, Clinic of Internal Medicine, Division of Medical \\ Oncology, Ankara, Turkey \\ ${ }^{4}$ University of Health Sciences Turkey, Gülhane Faculty of Medicine, Department of Internal Medicine, Ankara, Turkey \\ 5University of Health Sciences Turkey, Gülhane Faculty of Medicine, Department of Internal Medicine, Division of \\ Gastroenterology, Ankara, Turkey
}

Date submitted:

06.09.2019

Date accepted:

27.01.2020

Online publication date:

15.09.2020

\section{Corresponding Author:}

Sait Yeşillik, MD, University of Health Sciences Turkey, Gülhane

Training and Research Hospital,

Clinic of Internal Medicine, Division of Immunology and Allergy,

Ankara, Turkey

syesillik@yahoo.com

ORCID:

orcid.org/0000-0003-1160-1381

Keywords: Gastric cancer, cyclooxygenase-2, protein P21, gastritis, atrophic gastritis, intestinal metaplasia

\begin{abstract}
Aims: Early diagnosis and treatment can improve the prognosis of patients with gastric cancer. Therefore, identification and follow-up of patients who are under the risk of gastric cancer can improve the success of treatment and quality of life.

Methods: In our study, cyclooxygenase-2 (COX2) and P21 expressions of the patients with gastric adenocarcinoma and its precursor lesions atrophic gastritis and intestinal metaplasia were examined retrospectively with the immunohistochemical method.

Results: Forty patients were enrolled in each group. Grade and intensity of COX2 expressions were high in all three groups in accordance with the literature. In contrast with COX2 expressions, the grade and intensity of P21 expressions were very low in all three groups. Expressions of P21 were not observed in the vast majority of the gastric cancer group. Due to slight expressions, we detected statistically significant differences in both grading and intensity of P21 expressions between precancerous lesions and gastric cancer.
\end{abstract}

Conclusions: High COX2 expressions were consistent with literature. Decreased P21 expressions can reveal the relationship between loss of P21 expression and poor prognosis. Increased COX2, expression, especially in atrophic gastritis patients, and decreased P21 expression may be used in the follow-up of patients with gastric cancer and its precursor lesions.

\section{Introduction}

Although the incidence of gastric cancer decreases, it is still one of the most common causes of cancer mortality due to late diagnosis. Early diagnosis and treatment may be beneficial for preventing diseases, survival, and cost-effectiveness. Therefore, identification and follow-up of patients who are under the risk of gastric cancer increase the success of treatment.

Homeostasis of gastric mucosa is sustained by prostaglandins (1). Cyclooxygenase-2 (COX2) is expressed at gastric mucosa which is low in healthy tissue and high in the granulation tissue 
of gastric ulcus and gastric cancer (2,3). Also, COX2 expression is increased in Barret's metaplasia, dysplasia, and esophagus adenocarcinoma (4-6).

P21 gene is the primer mediator of P53 which induced cell apoptosis. Protein P21, encoded by this gene, inhibits G1 cyclin-dependent kinase and helps drive cells into $S$ phase (7). Czerniak et al. (8) reported P21 expression to be negative in almost all benign gastric and colonic lesions and positive in all malignant gastric and colonic lesions.

This study aims to determine the differences of COX2 and P21 expressions between patients with gastric cancer and its precursor lesions, atrophic gastritis and intestinal metaplasia.

\section{Methods}

In our study, pathology specimens of gastrectomy surgery and upper gastrointestinal endoscopy biopsies were assessed retrospectively. This study was conducted from June 2003 to December 2004. Anti-COX2 and anti-P21 were purchased from BD Biosciences (San Jose, CA, USA). Expressions of COX2 and P21 were evaluated in the pathology slides of patients with atrophic gastritis, intestinal metaplasia and gastric cancer (intestinal and diffuse type adenocarcinoma). 40 patients were enrolled for each group, and each pathology slide was labeled with COX2 and P21 antibodies separately, using immunohistochemical method. A total of 240 preparations were stained and evaluated.

Exclusion criteria included being under the age of 18 years, having another inflammatory, autoimmune rheumatic, infectious diseases and other types of cancers.

\section{Immunohistochemical Method}

Two independent pathologists made the evaluation. KS 400 software was used for grading and intensity. Each slide was evaluated with $100 \mathrm{X}$ magnification and visualized by KS 400 software on Microsoft R Windows NT 4.OR Service Pock 6.0 operating system. P21 and COX2 were marked for measurement on available sides. Positive and negative cells were marked by using KS 400 software and results were reported by software as percentages. P21 and COX2 positive cells stained brown (Figure 1).

COX2 and P21 expressions were evaluated in terms of grading, intensity, and immunoreactivity. Grade: Classified as $0,1(1-29 \%), 2(30-59 \%), 3(60-100 \%)$, according to involvement of COX2 and P21 antibodies percentage in the pathology slides.

Intensity: Classified as 0 (no involvement), 1 (mild involvement, diffuse cytoplasmic staining is less than $10 \%$ of cells), 2 (moderate involvement, strong granular cytoplasmic staining in $10-90 \%$ of cells), 3 (severe involvement, strong granular cytoplasmic staining in more than $90 \%$ of cells) according to power of involvement of COX2 and P21 antibodies in the pathology slides (9).

Immunoreactivity score: Calculated by the multiplication of grade and intensity (2).

\section{Statistical Analysis}

Commercially available software SPSS (version 21.0, SPSS Inc, Chicago, IL) was used for the statistical analyses. The chi-square test and one-way ANOVA test were used for the comparison. $\mathrm{P}<0.05$ was considered significant.

\section{Results}

A total of 120 patients ( $40 \%$ women) were enrolled with an average age of 64 years (25-87) in this study. There was no significant difference between the expression levels of men and women $(p=0.054)$. In this study, 19 of 40 gastric cancer patients were intestinal type, and 21 were diffuse type adenocarcinoma. Only 1 of the patients was early stage-gastric cancer.

\section{COX2 Expressions}

COX2 expression grades were observed to be high and similar in the three groups. Third-grade expression was detected in most of the lesions. There was no significant difference between COX2 expression grades $(p=0.06)$, (Figure 1$)$. No expression was detected in $3(2.5 \%)$ of 120 patients, $2(1.7 \%)$ of which were in atrophic gastritis group, and $1(0.8 \%)$ was in the gastric cancer group. Grade 3 expression was observed in thirty-two (26.7\%) patients with atrophic gastritis, $35(29.2 \%)$ with intestinal metaplasia and $25(20.8 \%)$ with gastric cancer (Table 1).

The strongest intensity of expressions was observed in the intestinal metaplasia and gastric cancer group, weaker intensity of expression was observed in the group with atrophic gastritis. There was a significant difference in COX2 intensity of expression between atrophic gastritis and gastric cancer $(p=0.026)$.

Table 1. Cyclooxygenase-2 expression grades and patient numbers

\begin{tabular}{llllll}
\hline Diseases & $\begin{array}{l}\text { Grade } \mathbf{0} \\
(\mathbf{0} \%)\end{array}$ & $\begin{array}{l}\text { Grade 1 } \\
(\mathbf{1 - 2 9 \% )}\end{array}$ & $\begin{array}{l}\text { Grade 2 } \\
(\mathbf{3 0 - 5 9 \% )}\end{array}$ & $\begin{array}{l}\text { Grade 3 } \\
(\mathbf{6 0 - 1 0 0 \% )}\end{array}$ & $\begin{array}{l}\text { Number of } \\
\text { patients }\end{array}$ \\
\hline Atrophic gastritis & $2(5)$ & $2(5)$ & $4(10)$ & $32(80)$ & $40(100)$ \\
\hline Intestinal metaplasia & $0(0)$ & $1(2.5)$ & $4(10)$ & $35(87.5)$ & $40(100)$ \\
\hline Gastric cancer & $1(2.5)$ & $8(20)$ & $6(15)$ & $25(62.5)$ & $40(100)$ \\
\hline Grade: Classified as 0, 1(1-29\%), 2(30-59\%), 3(60-100\%)
\end{tabular}


While strong expressions were detected in $3(2.5 \%)$ patients with atrophic gastritis, 9 (7.5\%) with intestinal metaplasia and 12 $(10 \%)$ with gastric cancer, there was no expression detected in $2(1.7 \%)$ with atrophic gastritis and $1(0.8 \%)$ with gastric cancer (Table 2).

The intensity of COX2 expression was detected similar between intestinal metaplasia and gastric cancer groups as $2^{\text {nd }}$ and $3^{\text {rd }}$ grade. COX2 expression was also detected in the atrophic gastritis group, but the intensity of expression was mostly $1^{\text {st }}$ and $2^{\text {nd }}$ grade. Same results were observed in the statistical analysis made with immunoreactivity scores.

When we evaluated immunoreactivity scores of COX2 expression between the groups, we found a significant difference between the groups with atrophic gastritis and intestinal metaplasia $(p=0.02)$, and also in the groups with atrophic gastritis and gastric cancer $(p=0.033)$. There was no significant difference between the groups with intestinal metaplasia and gastric cancer $(p=0.345)$.

\section{P21 Expressions}

There was a significant difference in grading and intensity of P21 expression between the atrophic gastritis, intestinal metaplasia, and gastric cancer groups $(p<0.001, p<0.001$, respectively) (Figure 1).

There was no expression in 61 (50.8\%) of 120 patients. Grade 1 expression was observed in the majority of atrophic gastritis, intestinal metaplasia, and gastric cancer patients. Grade 2 expression was observed in $3(2.5 \%)$ patients with atrophic gastritis, $3(2.5 \%)$ of the patients with intestinal metaplasia and $1(0.8 \%)$ of the patients with gastric cancer. Grade 3 expression was not observed in any patient.

The intensity of P21 expression was not observed in the vast majority of the gastric cancer group (90\%).

Grade 2 intensity of expression was observed in $1(0.8 \%)$ of the patients with atrophic gastritis, $6(5 \%)$ of the patients with intestinal metaplasia, and $2(1.7 \%)$ of the patients with gastric cancer. Grade 3 intensity of expression was not detected in any patient (Table 3,4).

Results of grading and intensity of P21 expression were found to be similar. When we evaluated immunoreactivity scores of P21 expression, we found it low in all three groups. Expression was not observed in the vast majority of the gastric cancer group. Because of slight expression in the

\begin{tabular}{|c|c|c|c|c|c|}
\hline Diseases & $\begin{array}{l}\text { Intensity } \\
0(\%)\end{array}$ & $\begin{array}{l}\text { Intensity } \\
1(\%)\end{array}$ & $\begin{array}{l}\text { Intensity } \\
2(\%)\end{array}$ & $\begin{array}{l}\text { Intensity } \\
3(\%)\end{array}$ & $\begin{array}{l}\text { Number of } \\
\text { patients (\%) }\end{array}$ \\
\hline Atrophic gastritis & $2(5)$ & $19(42.5)$ & $16(40)$ & $3(7.5)$ & $40(100)$ \\
\hline Intestinal metaplasia & $0(0)$ & $8(20)$ & $23(57.5)$ & 9 (22.5) & $40(100)$ \\
\hline Gastric cancer & $1(2.5)$ & $10(25)$ & $17(42.5)$ & $12(30)$ & $40(100)$ \\
\hline
\end{tabular}

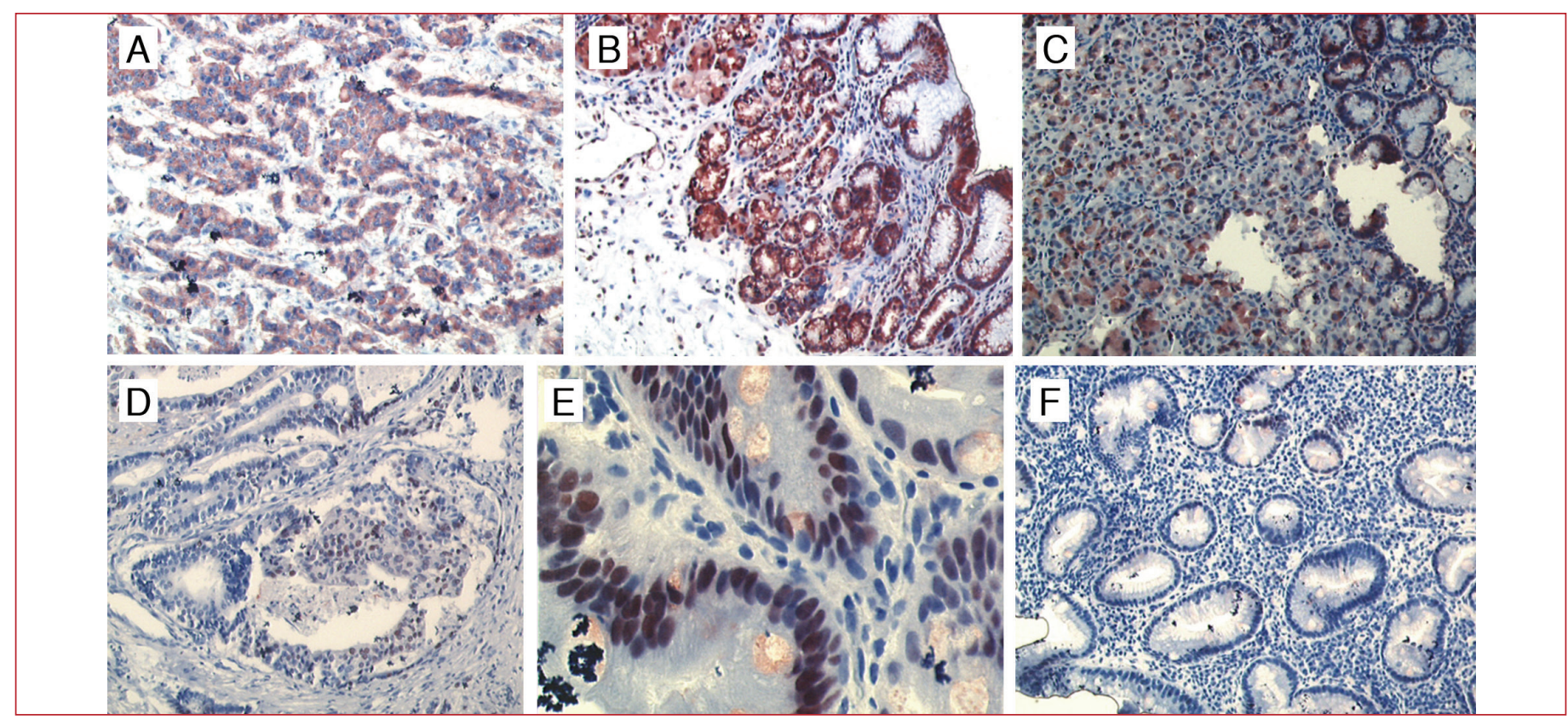

Figure 1. Cyclooxygenase-2 expression in (A) gastric cancer, (B) atrophic gastritis, (C) intestinal metaplasia, P21 expression in (D) gastric cancer, (E) atrophic gastritis, $(\mathrm{F})$ intestinal metaplasia 


\begin{tabular}{|c|c|c|c|c|c|}
\hline Diseases & $\begin{array}{l}\text { Grade } 0 \\
(0 \%)\end{array}$ & $\begin{array}{l}\text { Grade } 1 \\
(1-29 \%)\end{array}$ & $\begin{array}{l}\text { Grade } 2 \\
(30-59 \%)\end{array}$ & $\begin{array}{l}\text { Grade } 3 \\
(60-100 \%)\end{array}$ & $\begin{array}{l}\text { Number of } \\
\text { patients }\end{array}$ \\
\hline Atrophic gastritis & $13(32.5)$ & $24(60)$ & $3(7.5)$ & $0(0)$ & $40(100)$ \\
\hline Intestinal metaplasia & $12(30)$ & $25(62.5)$ & $3(7.5)$ & $0(0)$ & $40(100)$ \\
\hline Gastric cancer & $36(90)$ & $3(7.5)$ & $1(2.5)$ & $0(0)$ & $40(100)$ \\
\hline
\end{tabular}

\begin{tabular}{|c|c|c|c|c|c|}
\hline Diseases & $\begin{array}{l}\text { Intensity } \\
0(\%)\end{array}$ & $\begin{array}{l}\text { Intensity } \\
1(\%)\end{array}$ & $\begin{array}{l}\text { Intensity } \\
2(\%)\end{array}$ & $\begin{array}{l}\text { Intensity } \\
3(\%)\end{array}$ & $\begin{array}{l}\text { Number of } \\
\text { patients }\end{array}$ \\
\hline Atrophic gastritis & $13(32.5)$ & $26(65)$ & $1(2.5)$ & $0(0)$ & $40(100)$ \\
\hline Intestinal metaplasia & $12(30)$ & $22(55)$ & $6(15)$ & $0(0)$ & $40(100)$ \\
\hline Gastric cancer & $36(90)$ & $2(5)$ & $2(5)$ & $0(0)$ & 40 (100) \\
\hline
\end{tabular}

atrophic gastritis and intestinal metaplasia groups, there was a statistically significant difference between gastric cancer and these two groups $(p<0.001)$. There was no significant difference between the groups of intestinal metaplasia and atrophic gastritis $(p=0.339)$.

\section{Discussion}

In this study, COX2 and P21 expressions of the patients with gastric adenocarcinoma and its precursor lesions (atrophic gastritis and intestinal metaplasia) were evaluated. There was no significant difference in COX2 expression grades among the three groups. Grade 3 expression was seen in the vast majority of pathology slides in all groups. There was a significant difference in terms of COX2 expression intensity among the groups. Although COX2 expression was observed in all three groups, intensity was lower in the atrophic gastritis group. COX2 expression was more intense in the gastric cancer and intestinal metaplasia group. Especially increased COX2 expressions in atrophic gastritis patients can help doctors to predict cancer progression. It can be related to progress to gastric cancer. In our study, the increase in COX2 expression was found to be significant in gastric cancer and its precursor lesions, in accordance with the literature (26). In our study, the intensity of COX2 expression in lesions and lesion free healthy gastric mucosa, as a control group, was evaluated in each pathology slides. There was no significant difference between healthy and atrophic areas of gastric mucosa in patients with atrophic gastritis. Expression levels were similar. We evaluated that COX2 expression in healthy gastric mucosa might be a result of atrophy at the molecular level even if pathological atrophy did not occur yet. Helicobacter pylori (HP) infection might be included in this situation (10).
COX2 expressions were high in both gastric cancer and its precursor lesions. Thus, COX2 expression may be used in the follow-up of patients with gastric cancer and its precursor lesions. Increased levels of COX2 can help the doctors to follow up such patients, especially in cancer progression and metastasis.

When healthy gastric mucosa and intestinal metaplasia areas were compared in the intestinal metaplasia group, COX2 expression was observed in both areas. We thought that this might be a result of intestinal metaplasia started at the molecular level or might be a result of HP infection which is known to increase COX2 expression and very common in our society (10).

Lim et al. (2) found that COX2 expression was increased in intestinal metaplasia and gastric cancer areas while COX2 expression could be detected in healthy mucosa.

In the gastric cancer group, COX2 expression in healthy areas was statistically significantly lower than in areas with cancer. Shirvani et al. (5) also reported COX2 expression in Barrett's metaplasia and dysplasia was higher than healthy mucosa, as in our study.

We believe that this is because of the advanced cancer stages of our patients. Saukkonen et al. (11) demonstrated COX2 expression as $58 \%$ and $6 \%$ in intestinal metaplasia and early-stage gastric cancer patients, respectively. We found similar expressions in groups and it was $97.5 \%$. We thought that this was a result of 39 of 40 patients in our study who were in the advanced stage of cancer. In our study, COX2 expression in atrophic gastritis, intestinal metaplasia, and gastric cancer groups complies with literature.

We thought that increased COX2 expression was associated with inflammation, HP existence, growth factors, and cytokines besides cancer and could be beneficial in precursor lesions to follow up. 
Rossolymos et al. (12) detected that increased COX2 expression scores were associated with the severity of gastritis, despite our positive findings, he found a weak correlation between COX2 expression scores and precancerous lesions like atrophic gastritis and intestinal metaplasia.

We also investigated the significant difference in P21 expression grade between healthy and atrophic areas of gastric mucosa in the atrophic gastric group. Probably due to the inability of P21 expression in atrophic cells, P21 expression was weakly increased as a result of atrophy at the molecular level in healthy tissue. Wang et al. (13) studied P21 expression in patients with chronic gastritis, atrophic gastritis, intestinal metaplasia, early-stage, and advanced gastric cancer. In this study, similar to our findings, P21 expression was found as $40 \%$ in HP (-) healthy mucosa areas, $10 \%$ in atrophic gastritis areas, $60 \%$ in intestinal metaplasia areas, $33 \%$ in nonneoplastic areas adjacent to early gastric cancer and $23 \%$ in nonneoplastic areas adjacent to advanced gastric cancer. P21 expressions in intestinal metaplasia and advanced gastric cancer areas of gastric mucosa were weak and similar. Despite the increase in COX2 expression, the loss in expression of P21 can be a predictor of cancer progression and metastasis.

Sun et al. (14) determined P21 expression in healthy areas of gastric mucosa. Noguchi et al. (15) showed P21 expression in intestinal metaplasia areas, and they failed to show P21 expression in healthy areas.

In our study, there was no P21 expression determined in almost all healthy and gastric cancer areas. While these findings in the normal tissue correlate with present literature, they are not in accordance with the gastric cancer tissue $(8,16)$.

In our study, P21 expression was observed in $49.2 \%$ of 120 patients. In the majority of literature findings, lowest grade and intensity of expressions were demonstrated in our gastric cancer group (10\%). We thought this was a result of that only one of the patients was early gastric cancer, 39 patients were advanced gastric cancer because many studies in literature showed that loss of P21 expression was a finding of advanced cancer and metastasis (17-20).

Grade and intensity of P21 expression were low and similar in atrophic gastritis and intestinal metaplasia groups. While Gamboa-Dominguez (21) and Kouraklis et al. (22) found a positive correlation between P21 expression and survival, other studies showed that the loss of P21 expression in cancer was associated with poor prognosis $(18,23)$.

P21 expression was studied by Sun et al. (14) on 139 patients (42 incomplete metaplasia, 28 low-grade dysplasia, 21 high-grade dysplasia, 48 intestinal-type gastric cancer). They found P21 expression in some of the normal mucosa, similar to our findings. Although it was statistically insignificant, P21 expression demonstrated a reduction from incomplete metaplasia towards gastric cancer (incomplete metaplasia $47.6 \%$, low-grade dysplasia 39.3\%, high-grade dysplasia $33.3 \%$, intestinal-type gastric cancer $29.5 \%$ ). With these results, Sun stated that a gradual decrease observed in P21 expression might be significant in the progression of cancer.

In our study, the percentage and the intensity of P21 expression in gastric cancer were not in accordance with some of the literature $(8,16)$. On the other hand, the studies that suggest the loss of P21 expression to be the cause of poor prognosis (14,17-23) support the low P21 expressions in our study.

Like in the expression of COX2 (the expression was significantly high), we thought this difference might be due to the fact that only one of our gastric cancer patient was early stage gastric cancer and the others were advanced gastric cancer. As it has been indicated before, multifactorial factors such as the organ, stage, immunohistochemistry and the molecular mechanism are all sufficient for the decision (24). It is possible to suggest that molecules such as COX2 expression and loss of P21 expression may be related to gastric cancer and poor outcome.

The limitation of our study is that if we could able to study consecutively P21 and COX2 expressions in the same patients with precursor lesions and gastric cancer, the results might be more significant. Our study was a retrospective study. Prospective researches with a larger number of patients attending and supported by genetic and experimental studies in the future will be able to demonstrate the importance of COX2 and P21 expressions in gastric cancer and precursor lesions more clearly. Also, in our study, most of the gastric patients were late diagnosed. We could have found different results if we had been able to study with early diagnosed gastric cancer patients.

\section{Conclusion}

To the best of our knowledge, this has been the first study evaluating both COX2 and P21 expressions in gastric cancer and its precursor lesions of atrophic gastritis and intestinal metaplasia.

In the future, COX2 expression may be used in the follow-up of patients with gastric cancer and atrophic gastritis. The loss of P21 expression may indicate poor prognosis and progression in gastric cancer.

\section{Ethics}

Ethics Committee Approval: This research was approved by Gülhane Training and Research Hospital Scientific Research Board, Ankara (AR-2004/54). During the time this study was conducted, retrospective studies were not subject to institutional review board approval. 
Informed Consent: This research was a retrospective study.

Peer-review: Externally peer-reviewed.

\section{Authorship Contributions}

Surgical and Medical Practices: M.G., Concept: M.G., S.Y., Design: M.G., S.Y., Data Collection or Processing: S.Y., M.A., Analysis or Interpretation: M.A., M.G., S.Y., Literature Search: S.Y., B.Y., Writing: S.Y., B.B.B.

Conflict of Interest: No conflict of interest was declared by the authors.

Financial Disclosure: The authors declared that this study received financial support from Gülhane Training and Research Hospital Scientific Research Board, Ankara (AR-2004/54).

\section{References}

1. Eberhart CE, Dubois RN. Eicosanoids and the gastrointestinal tract. Gastroenterology. 1995;109:285301.

2. Lim HY, Joo HJ, Choi JH, et al. Increased expression of cycloxygenase-2 protein in human gastric carcinoma. Clin Cancer Res. 2000;6:519-525.

3. Mizuno $\mathrm{H}$, Sakomoto $\mathrm{C}$, Matsuda $\mathrm{K}$, et al. Induction of cyclooxygenase-2 in gastric mucosal lesions and its inhibition by the specific antagonist delays healing mice. Gastroenterology. 1997;112:387-397.

4. Van Rees BP, Saukkonen K, Ristimaki A, et al. Cyclooxygenase-2 expression during carcinogenesis in the human stomach. J Pathol. 2002;196:171-179.

5. Shirvani VN, Ouatu-Lascar R, Kaur BS, Omary MB, Triadafilopoulos G. yclooxygenase 2 expression in Barrett's esophagus and adenocarcinoma: Ex vivo induction by bile salts and acid exposure. Gastroenterology. 2000;118:487496.

6. Türkmen IÇ, Başsüllü N, Uraz S, Yerdel MA, Memışoğlu R, Bülbül Doğusoy G. CDX2, COX2 and MUC2 expressions in Barrett's esophagus; Can they be useful in determination of the dysplasia. Turk Patoloji Derg. 2012;28:251-258.

7. Harper JW, Adami GR, Wei N, Keyomarsi K, Elledge SJ. The P21, interacting protein Cip 1 is a potent inhibitor of G1 cyclin-dependent kinases. Cell. 1993;75:805-816.

8. Czerniak B, Herz F, Koss LG, Schlom J. Ras oncogene as P21 as a tumor marker in cytodiagnosis of gastric and colonic carcinomas. Cancer. 1987;60:2432-2436.

9. Ristimaki A, Sivula A, Ludin J, et al. Prognostic significance of elevated cyclooxygenase-2 expression in breast cancer. Cancer Res. 2002;62:632-635.

10. Kimura A, Tsuji S, Tsujii M, et al. Expression of COX2 and nitrotyrosine in human gastric mucosa before and after Helicobacter Pylori eradication. Prostaglandins Leukot Essent Fatty Acids. 2000;63:315-322.
11. Saukkonen K, Nieminen O, Van Rees B, et al. Expression of COX2 in the dysplasia of the stomach and in intestinal type gastric adenocarcinoma. Clin Cancer Res. 2001;7:19231931.

12. Rossolymos NA, Papatheodoridis G, Tsakoniatis $M$, Dimitrios K, Lazaris A. Expression of cyclooxygenase-2 in gastritis and gastric premalignant lesions. Ann Gastroenterol. 2013;26:275.

13. Wang J, Chi DS, Kalin GB, et al. Helicobacter Pylori infection and oncogene expressions in gastric cancer and its precursor lesions. Dig Dis Sci. 2002;47:107-113.

14. Sun Y, Li JY, He JS, Zhou LX, Chen K. Tissue microarray analysis of multiple gene expression in intestinal metaplasia, dysplasia, and carcinoma of the stomach. Histopathology. 2005;46:505-514.

15. Noguchi M, Hirohashi S, Shimosato $Y$, et al. Histologic demonstration of antigens reactive with anti-P21 Ras monoclonal antibody (RAP-5) in human stomach Cancers. J Natl Cancer Inst. 1986;77:379-385.

16. Craanen ME, Blok P, Offerhaus GJ, et al. P21(Waf/Cip1) expression and P53/MDM2 feedback loop in gastric carcinogenesis. J Pathol. 1999;189:481-486.

17. Nomura A, Stemmermann GN, Chyou PH, Kato I, PerezPerez GI, Blaser MJ. Helicobacter Pylori infection and gastric carcinoma among Japanese Americans in Hawaii. N Engl J Med. 1991;325:1132-1136.

18. Ogawa M, Maeda K, Onoda N, Chung YS, Sowa M. Loss of p21WAF1/CIP1 expression correlates with disease progression in gastric carcinoma. $\mathrm{Br} \mathrm{J}$ Cancer. 1997;5:1617-1620.

19. Liu X, Yu H, Cai H, Wang Y. Expression of CD24, p21, p53, and c-myc in alpha-fetoprotein producing gastric cancer: correlation with clinicopathologic characteristics and survival. J Surg Oncol. 2014;109:859-864.

20. Gunia S, Kakies C, Erbersdobler A, Hakenberg OW, Koch S, May M. Expression of p53, p21 and cyclin D1 in penile cancer: p53 predicts poor prognosis. J Clin Pathol. 2012;65:232-236.

21. Gamboa-Dominguez A, Seidl S, Reyes-Gutierrez E, et al. Prognostic significance of p21WAF1/CIP1, p27Kip1, p53 and Ecadherin expression in gastric cancer. J Clin Pathol. 2007;60:756-761.

22. Kouraklis G, Katsoulis IE, Theocharis S, et al. Does the expression of cyclin E, pRb, and p21 correlate with prognosis in gastric adenocarcinoma? Dig Dis Sci. 2009;54:1015-1020.

23. Ozen A, Kocak Z, Sipahi T, et al. The prognostic significance of p21 and Her2 gene expression and mutation/ polymorphism in patients with gastric adenocarcinoma. Med Oncol. 2013;30:357-362.

24. Karadurmuş N, Ertürk İ. Which is the best for cancer treatment? Surgery or chemotherapy. J Oncol Sci. 2017;3:32-33. 